\section{Transmit-Diversity-Assisted Space-Shift Keying for Colocated and Distributed/Cooperative MIMO Elements}

\author{
Du Yang, Chao Xu, Student Member, IEEE, \\ Lie-Liang Yang, Senior Member, IEEE, and \\ Lajos Hanzo, Fellow, IEEE
}

\begin{abstract}
Space-shift keying (SSK) modulation is a recently proposed multiple-input-multiple-output (MIMO) technique, which activates only a single transmit antenna during each time slot and uses the specific index of the activated transmit antenna to implicitly convey information. Activating a single antenna is beneficial in terms of eliminating the interchannel interference and mitigates the peak-to-mean power ratio while avoiding the need for synchronization among transmit antennas. However, this benefit is achieved at a sacrifice, because the transmit diversity gain potential of the multiple transmit antennas is not fully exploited in existing SSK-assisted systems. Furthermore, a high-SSK throughput requires the transmitter to employ a high number of transmit antennas, which is not always practical. Hence, we propose four algorithms - open-loop space-time space-shift keying (ST-SSK), closed-loop feedback-aided phase rotation, feedback-aided power allocation, and cooperative ST-SSK-to achieve a diversity gain. The performance improvements of the proposed schemes are demonstrated by Monte Carlo simulations for spatially independent Rayleigh fading channels. Their robustness to channel estimation errors is also considered. We advocate the proposed ST-SSK techniques, which can achieve a transmit diversity gain of about $10 \mathrm{~dB}$ at a bit error rate (BER) of $10^{-5}$, at the cost of imposing a moderate throughput loss that is dedicated to a modest feedback overhead. Furthermore, our proposed ST-SSK scheme lends itself to efficient communication, because the deleterious effects of deep shadow fading no longer impose spatial correlation on the signals that are received by the antennas, which cannot readily be avoided by colocated antenna elements.
\end{abstract}

Index Terms-Amplify and forward (AF), feedback, phase rotation, power allocation, space-shift keying (SSK), space-time, transmit diversity.

\section{INTRODUCTION}

Space-shift keying (SSK) modulation, which uses the specific index of the activated transmit antennas to implicitly convey information, was proposed by Jeganathan et al. [1]. This idea may be traced back to [2], which was then further developed into spatial modulation (SM) by Mesleh and Haas [3], [4]. Compared with the conventional multipleinput-multiple-output (MIMO) schemes [5], e.g., space-time block coding (STBC) [6], the Vertical Bell Laboratories Layered SpaceTime (V-BLAST) scheme [5], spatial-division multiple access (SDMA) [7], and beamforming [8], SSK modulation activates only a single transmit antenna at each symbol instant and has the following advantages.

1) It avoids synchronization among transmit antennas, because only a single antenna is activated during a time slot. This feature

Manuscript received August 26, 2010; revised November 23, 2010, February 10, 2011, and April 18, 2011; accepted May 12, 2011. Date of publication June 13, 2011; date of current version July 18, 2011. This work was supported in part by the Research Councils U.K. through the India-U.K. Advanced Technology Centre, by the Engineering and Physical Sciences Research Council through the China-U.K. Science Bridge, and by the European Union through the CONCERTO Project. The review of this paper was coordinated by Dr. D. W. Matolak.

The authors are with the School of Electronics and Computer Science, University of Southampton, SO17 1BJ Southampton, U.K. (e-mail: dy05r@ ecs.soton.ac.uk; cx1g08@ecs.soton.ac.uk; 1ly@ecs.soton.ac.uk; 1h@ecs.soton. ac.uk).

Color versions of one or more of the figures in this paper are available online at http://ieeexplore.ieee.org.

Digital Object Identifier 10.1109/TVT.2011.2157185 can particularly be useful for communication systems that rely on distributed antennas in cooperative communication.

2) It circumvents the spatial antenna correlation that is imposed by the deleterious effects of deep shadow fading, which cannot readily be avoided by the classic STBC schemes using colocated antenna elements.

3) It eliminates the interchannel interference among transmit antennas, mitigates the peak-to-mean envelope ratio, and achieves full receiver diversity through low-complexity single-stream [1] rather than multistream receivers [9], [10].

4) It is robust to channel estimation errors at the receiver side [1], because it is not the actual channel realization but the differences between channels associated with the different transmit antennas, which determines the bit-error-rate (BER) performance.

The novel contribution of this paper is that we proposed the following three algorithms to achieve transmit diversity for SSK modulation: 1) an open-loop space-time space-shift keying (ST-SSK) scheme; 2) a feedback-aided phase rotation scheme, and 3) another closedloop scheme that uses feedback-aided power allocation. ${ }^{1}$ Furthermore, we proposed an amplify-and-forward (AF)-relaying-aided cooperative ST-SSK scheme to avoid the employment of a high number of transmit antennas at the cooperating nodes, which allowed us to mitigate the potential performance degradation caused by deep shadow fading.

This paper is organized as follows. The original SSK scheme in [1] is briefly introduced in Section II. The proposed transmit-diversityassisted SSK modulation schemes are detailed in Section III. Our simulation results are provided in Section IV, followed by our conclusions in Section V.

\section{Preliminaries: SpaCe-Shift Keying Modulation}

Without any loss of generality, we will focus our attention on the $\left(N_{T x} \times 1\right)$-element MISO systems, which are illustrated in Fig. 1 . The extension of our results to systems with more than one receive antenna is straightforward. A random sequence of independent bits $\boldsymbol{a}=\left[a_{1}, \ldots, a_{N_{a}}\right]$ enters the channel encoder, which produces the vector $c=\left[c_{1}, \ldots, c_{N_{c}}\right]$, where $N_{a}$ and $N_{c}$ represent the number of channel encoder input and output bits, respectively. The resulting coding rate $r_{c}$ becomes $r_{c}=N_{a} / N_{c}$. The pseudo randomly interleaved encoded bits $\Pi(c)$ then enter an SSK bit-to-symbol mapper. A group of $m=\log _{2} N_{T x}$ bits is then mapped to a constellation vector $\boldsymbol{x}$ with only a single nonzero element $\sqrt{m E_{b}}$ at the $j$ th position, which is formulated as

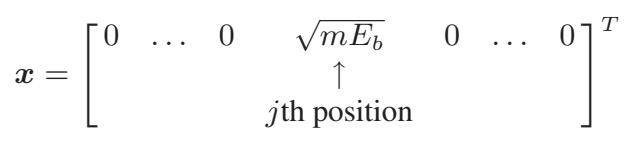

where $E_{b}$ represents the average energy of a single bit. Although the symbol $x_{j}=\sqrt{m E_{b}}$ does not contain any information, its location does. Hence, only the $j$ th transmit antenna will be activated for transmission. The modulated signal is then transmitted over the $N_{T x^{-}}$ element wireless channel $\boldsymbol{h}$, which is contaminated by the additive white Gaussian noise (AWGN) $n \sim \mathcal{C N}\left(0, N_{0}\right)$, where $N_{0}$ represents the noise variance. The received signal is given by $y=\boldsymbol{h} \boldsymbol{x}+n$.

\footnotetext{
${ }^{1}$ The opportunistic power-allocation-assisted SSK proposed for systems with two transmit antennas in [11] may be considered a special case of our scheme.
} 


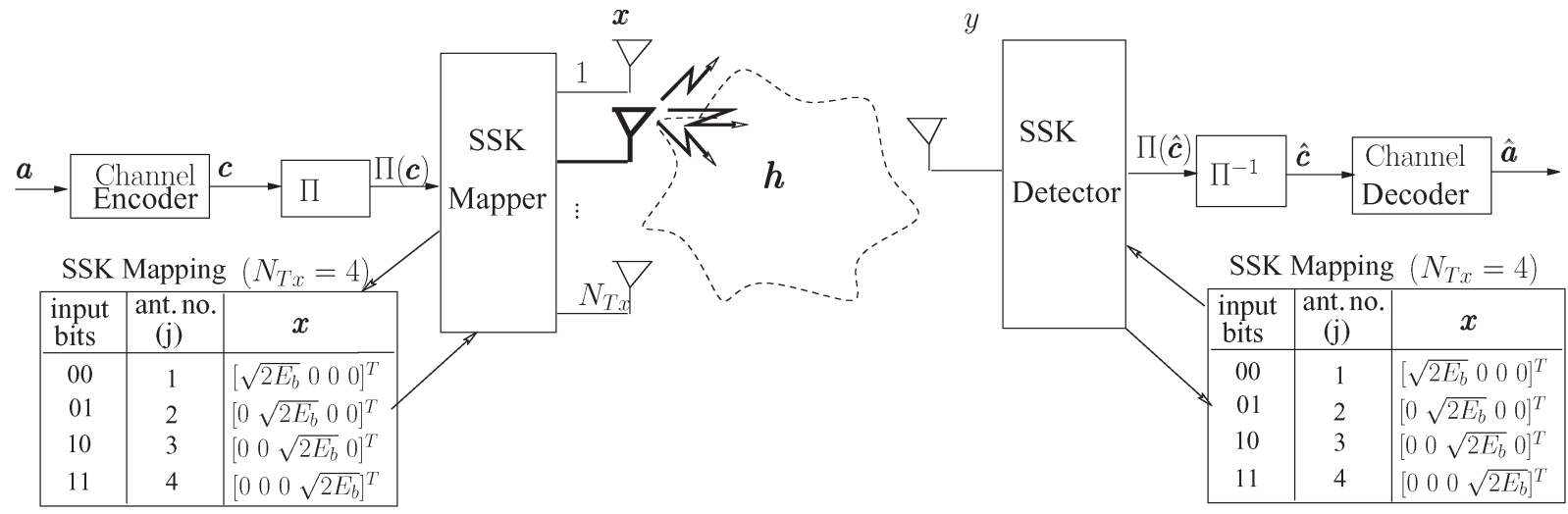

Fig. 1. SSK-modulation-aided MISO system with $N_{T x}$ transmit and a single receiver antenna.

Assuming that the channel vector $\boldsymbol{h}$ is perfectly known, the index of the activated antenna $\hat{j}$ is determined by [12]

$$
\begin{aligned}
\hat{j} & =\arg \min _{j}\left(\left|y-\sqrt{m E_{b}} h_{j}\right|^{2}\right) \\
& =\arg \max _{j}\left(2 \sqrt{m E_{b}} \Re\left(h_{j}^{H} y\right)-m E_{b}\left|h_{i}\right|^{2}\right) .
\end{aligned}
$$

Given the knowledge of the SSK bit-to-symbol mapping rules, the transmitted information sequence is estimated and then correspondingly deinterleaved and decoded.

\section{TRAnsmit-Diversity-Assisted SPACE-ShifT KEYING MODULATION}

\section{A. Open Loop: ST-SSK for $N_{T x}>2$}

The proposed ST-SSK scheme uses $L$ time slots to transmit $m=\log _{2} N_{T x}$ bit sequences $\boldsymbol{a}_{i}$. At the $l$ th $(1 \leq l \leq L)$ time slot, the $f\left(t_{l}, \boldsymbol{a}_{i}\right)$ th $\left(1 \leq f\left(t_{l}, \boldsymbol{a}_{i}\right) \leq N_{T x}\right)$ transmit antenna is activated according to the information sequence $\boldsymbol{a}_{i}$, which is formulated as

$$
\left[\begin{array}{c}
y^{t_{1}} \\
\vdots \\
y^{t_{l}} \\
\vdots \\
y^{t_{L}}
\end{array}\right]=\sqrt{\frac{m E_{b}}{L}}\left[\begin{array}{c}
h_{f\left(t_{1}, \boldsymbol{a}_{i}\right)} \\
\vdots \\
h_{f\left(t_{l}, \boldsymbol{a}_{i}\right)} \\
\vdots \\
h_{f\left(t_{L}, \boldsymbol{a}_{i}\right)}
\end{array}\right]+\left[\begin{array}{c}
n^{t_{1}} \\
\vdots \\
n^{t_{l}} \\
\vdots \\
n^{t_{L}}
\end{array}\right] .
$$

Upon defining $\boldsymbol{y}=\left[y^{t_{1}}, \ldots, y^{t_{L}}\right]^{T}$ and $\boldsymbol{h}_{\boldsymbol{f}\left(\boldsymbol{a}_{i}\right)}=\left[h_{f\left(t_{1}, \boldsymbol{a}_{i}\right)}, \ldots\right.$, $\left.h_{f\left(t_{L}, a_{i}\right)}\right]^{T}$, the transmitted sequence $\hat{\boldsymbol{a}}_{i}$ is determined at the receiver side according to

$$
\begin{aligned}
\hat{\boldsymbol{a}}_{i} & =\arg \min _{\boldsymbol{a}_{i}}\left\|\boldsymbol{y}-\sqrt{\frac{m E_{b}}{L}} \boldsymbol{h}_{\boldsymbol{f}\left(\boldsymbol{a}_{i}\right)}\right\|_{2}^{2} \\
& =\arg \min _{\boldsymbol{a}_{i}} \sum_{l}^{L}\left|y_{t_{l}}-\sqrt{\frac{m E_{b}}{L}} h_{f\left(t_{l}, \boldsymbol{a}_{i}\right)}\right|^{2} .
\end{aligned}
$$

Before considering the design of the bit-to-antenna mapping function $f\left(t_{l}, \boldsymbol{a}_{i}\right)$, let us first prove the following two propositions.

Proposition 1: For an $\left(N_{T x} \times 1\right)$ ST-SSK-modulation-aided system that uses $L$ time slots, the transmit diversity order of $L$ is achieved if and only if, for any two bit sequences $\boldsymbol{a}_{i}$ and $\boldsymbol{a}_{j}(i \neq j)$ and for any two time instances $l$ and $n(l \neq n)$, the following conditions are satisfied.

1) The indices of the antennas that are activated to signal the bit sequence $\boldsymbol{a}_{i}$ and $\boldsymbol{a}_{j}$ are different from each other at any time slot $l$, which is formulated as $f\left(t_{l}, \boldsymbol{a}_{i}\right) \neq f\left(t_{l}, \boldsymbol{a}_{j}\right)$.

2) When transmitting $\boldsymbol{a}_{i}$ and $\boldsymbol{a}_{j}$, the difference between the activated channels' channel impulse response (CIR) taps at the $l$ th time slot, which is denoted by $\Delta h_{t_{l}}=h_{f\left(t_{l}, a_{i}\right)}-h_{f\left(t_{l}, a_{j}\right)}$, must be a random variable, which is independent of the CIR taps during the $n$th time slot, that is represented as $\Delta h_{t_{n}}=$ $h_{f\left(t_{n}, \boldsymbol{a}_{i}\right)}-h_{f\left(t_{n}, \boldsymbol{a}_{j}\right)}$.

Proof: Transmitting the bit sequences $\boldsymbol{a}_{i}$ and $\boldsymbol{a}_{j}(i \neq j)$, the Euclidean distance between the corresponding received signals without noise is formulated as

$$
\begin{aligned}
d & =\sqrt{\frac{m E_{b}}{L}}\left\|\boldsymbol{h}_{\boldsymbol{f}\left(\boldsymbol{a}_{i}\right)}-\boldsymbol{h}_{\boldsymbol{f}\left(\boldsymbol{a}_{j}\right)}\right\|_{F} \\
& =\sqrt{\frac{m E_{b}}{L} \sum_{l}^{L}\left|h_{f\left(t_{l}, \boldsymbol{a}_{i}\right)}-h_{f\left(t_{l}, \boldsymbol{a}_{j}\right)}\right|^{2}} \\
& =\sqrt{\frac{m E_{b}}{L} \sum_{l}^{L}\left|\Delta h_{t_{l}}\right|^{2} .}
\end{aligned}
$$

A diversity order of $L$ is achieved if and only if $\Delta h_{t_{l}}, l \in[1, L]$ are independent complex-valued Gaussian variables. As a result, $\forall i \neq j$, $l \neq n, i, j \in\left[1, N_{T x}\right], l, n \in[1, L]$, we have $f\left(t_{l}, \boldsymbol{a}_{i}\right) \neq f\left(t_{l}, \boldsymbol{a}_{j}\right)$, and $\left|h_{f\left(t_{l}, a_{i}\right)}-h_{f\left(t_{l}, a_{j}\right)}\right|$ must be independent of $\mid h_{f\left(t_{n}, a_{i}\right)}-$ $h_{f\left(t_{m}, a_{j}\right)} \mid$.

Proposition 2: For an $\left(N_{T x} \times 1\right)$ ST-SSK-modulation-aided system, the maximum achievable transmit diversity order cannot be higher than $\left(N_{T x}-1\right)$.

Proof: Assume that we have $L=N_{T x}$ when a transmit diversity order of $N_{T x}$ is achieved. According to Proposition 1, the Euclidean distance $d$ between $\boldsymbol{a}_{i}$ and $\boldsymbol{a}_{j}$ is a sum of $N_{T x}$ independent $\Delta h_{t_{l}}$ values. However, for $N_{T x}$ transmit antennas, there are at most $\left(N_{T x}-\right.$ 1 ) independent $\Delta h$ values. Hence, achieving a diversity order that is higher than $\left(N_{T x}-1\right)$ becomes impossible.

Let us now consider the design of the bit-to-antenna mapping function. We propose a shift-mapping function $f_{s}\left(t_{l}, \boldsymbol{a}_{i}\right)$ for scenarios with an even number of time slots and propose what we refer to as a shift-swap-mapping function $f_{s-w}\left(t_{l}, \boldsymbol{a}_{i}\right)$ for scenarios with an odd 
TABLE I

EXAmple of the Activated Transmit Antenna When (a) $N_{T x}=3$, $N_{R x}=1$ Using SHIFT BIT-TO-ANTENNA MAPPING $f_{s}\left(l, \boldsymbol{a}_{i}\right)$ AND

(b) $N_{T x}=4, N_{R x}=1$ Using SHIFT-SwAP BIT-TO-ANTENNA MAPPING $f_{s-w}\left(l, \boldsymbol{a}_{i}\right)$ AND THE SHIFT MAPPING $f_{s}\left(l, \boldsymbol{a}_{i}\right)$

(a)

\begin{tabular}{|c|c|c|}
\hline Input bits $\boldsymbol{a}_{i}$ & \multicolumn{2}{|c|}{ Activated antenna index $f_{s}\left(t_{l}, \boldsymbol{a}_{i}\right)$} \\
\cline { 2 - 3 } & $t_{1}$ & $t_{2}$ \\
\hline 0 & 1 & 2 \\
\hline 1 & 2 & 3 \\
\hline
\end{tabular}

(b)

\begin{tabular}{|c|c|c|c|}
\hline Input bits $\boldsymbol{a}_{i}$ & \multicolumn{3}{|c|}{$\begin{array}{c}\text { Activated antenna index } \\
f_{s-w}\left(t_{l}, \boldsymbol{a}_{i}\right)\left(f_{s}\left(t_{l}, \boldsymbol{a}_{i}\right)\right)\end{array}$} \\
\hline & $t_{1}$ & $t_{2}$ & $t_{3}$ \\
\hline 00 & $1(1)$ & $2(2)$ & $3(3)$ \\
\hline 01 & $2(2)$ & $3(3)$ & $4(4)$ \\
\hline 10 & $4(3)$ & $1(4)$ & $1(1)$ \\
\hline 11 & $3(4)$ & $4(1)$ & $2(2)$ \\
\hline
\end{tabular}

number of time slots. More explicitly, the algorithms are described as follows.

1) $f_{s}\left(t_{l}, \boldsymbol{a}_{i}\right)$ when $N_{T x}>2$ and $L$ is an even number.

- Set $\boldsymbol{v}=\left[1,2, \ldots, N_{T x}\right]$.

- Set $\left[f\left(t_{l}, \boldsymbol{a}_{1}\right), f\left(t_{l}, \boldsymbol{a}_{2}\right), \ldots, f\left(t_{l}, \boldsymbol{a}_{N_{T x}}\right)\right]=\operatorname{Shift}(\boldsymbol{v}, l-$ $1)$, where $\operatorname{Shift}(\boldsymbol{v}, l-1)$ represents a left circular shift of $(l-1)$ positions.

2) $f_{s-w}\left(t_{l}, \boldsymbol{a}_{i}\right)$ when $N_{T x}>2$ and $L$ is an odd number.

- Create a shift-mapping function following the aforementioned steps.

- Randomly group the last $\left(N_{T x}\right) / 2$ bit sequences $\left(\boldsymbol{a}_{\left(N_{T x} / 2\right)+1}, \ldots, \boldsymbol{a}_{N_{T x}}\right)$ into $\left(N_{T x}\right) / 4$ pairs.

- Within each pair, e.g., $\boldsymbol{a}_{\left(N_{T x} / 2\right)+1}$ and $\boldsymbol{a}_{\left(N_{T x} / 2\right)+2}$ grouped as a pair, swap the mapping results of $f\left(t_{l}, \boldsymbol{a}_{\left(N_{T x} / 2\right)+1}\right)$ and $f\left(t_{l}, \boldsymbol{a}_{\left(N_{T x} / 2\right)+2}\right)$ for all $l \leq$ $\left(N_{T x} / 2\right)$.

The examples of the activated transmit antenna index for $N_{T x}=3$ using the shift mapping of $f_{s}\left(t_{l}, \boldsymbol{a}_{i}\right)$ and for $N_{T x}=4$ using the shift-swap bit-to-antenna mapping of $f_{s-w}\left(t_{l}, \boldsymbol{a}_{i}\right)$ are shown in Table I, respectively. The shift-mapping results for $N_{T x}=4$ are also shown in Table I within the brackets. The proposed bit-to-antenna mapping functions have guaranteed that the transmit diversity order of $L$ is achieved if we have $L \leq\left(N_{T x}-1\right)$.

The proposed ST-SSK scheme may be modeled using the same structure as the orthogonal STBC [13] or the unitary space-time modulation [14], [15], which is given by the product of the channel matrix and an $\left(N_{T x} \times L\right)$-element coding matrix denoted as $\boldsymbol{C}$. However, for our ST-SSK scheme, every column vector of $\boldsymbol{C}$ contains only a single nonzero element, which is different from the other two schemes. More importantly, the coding matrix $C$ that is conceived for our ST-SSK scheme varies according to the input information vector $\boldsymbol{a}_{i}$. By contrast, for the other two schemes, the coding matrix is constant and is designed offline. As a result, although the ST-SSK scheme is inferior to the conventional STBC schemes in terms of throughput, it retains the beneficial properties of the original SSK modulation, e.g., low complexity that is achieved in the absence of interantenna synchronization, which is beneficial in cooperative/distributed MIMOs, as mentioned in the Introduction.

\section{B. Closed Loop: Feedback-Aided Phase Rotation for $N_{T x}=2$}

Based on Proposition 2, it is now plausible that, using the open-loop ST-SSK modulation scheme proposed in Section III-A, we can only achieve a transmit diversity order of one for $N_{T x}=2$, because there is only an $N_{T x}-1=1$ independent $\Delta h$ value. In this section, we propose a scheme that is particularly for $N_{T x}=2$, which feeds back a phase value $\theta$ from the receiver to the transmitter to maximize the Euclidean distance $d_{\text {min }}=d=\sqrt{E_{b}}\left\|\boldsymbol{h}_{1}-\boldsymbol{h}_{2}\right\|$ and, hence, achieve transmit diversity. The objective function that is used for our design may be written as

$$
\begin{aligned}
\arg \max _{\theta} d_{\text {min }}^{2} & =\arg \max _{\theta}\left\|h_{1}-h_{2} e^{j \theta}\right\|^{2} \\
& =\arg \max _{\theta}\left\|h_{1}\right\|^{2}+\left\|h_{2}\right\|^{2}-2 \Re\left(h_{1}^{H} h_{2} e^{j \theta}\right) \\
& =\arg \min _{\theta} \Re\left(h_{1}^{H} h_{2} e^{j \theta}\right) \\
& =\arg \min _{\theta} \Re\left(\left|h_{1}^{H} h_{2}\right| e^{j(\theta+\phi)}\right)
\end{aligned}
$$

where we have $h_{1}^{H} h_{2}=\left|h_{1}^{H} h_{2}\right| e^{j \phi}$. Explicitly, the minimum of (6) is achieved for

$$
\theta+\phi=(2 k+1) \pi, k \in \mathcal{Z} .
$$

As a result, the Euclidean distance becomes $d=\sqrt{E_{b}}\left(\left|h_{1}\right|+\left|h_{2}\right|\right)$ due to having two independent channel gains. Hence, a transmit diversity order of two is achieved. In fact, as long as we have $\Re\left(h_{1}^{H} h_{2} e^{j \theta}\right)>0$, a diversity order of two can be guaranteed. Moreover, $\theta$ is expected to have a uniform distribution between 0 and $2 \pi$. A scalar quantizer with uniformly distributed quantization levels constitutes an adequate yet low-complexity quantizer design.

\section{Closed Loop: Feedback-Aided Power Allocation}

Our proposed feedback-aided power allocation scheme is detailed as follows. First, the channel's fading coefficients $\left|h_{j}\right|$ associated with different transmit antennas are estimated at the receiver side. Second, the transmit antenna with the lowest channel gain is identified at the receiver, and its index is fed back to the transmitter using $\log _{2} N_{T x}$ bits. Upon receiving the feedback information, the transmitter will allocate zero power to the antenna with the lowest channel gain and increase the power that is allocated to other antennas from a value of $m E_{b}$ to $m E_{b}+\left(m E_{b} / N_{T x}-1\right)$. For example, two bits [0 0 ] will be fed back to the transmitter if we have $\left|h_{1}\right|<\left|h_{i}\right|, 1<i \leq 4$ for a system with four transmit antennas and a single receive antenna. The power that is allocated to the first transmit antenna is zero, whereas the power for the other antennas is increased from $2 E_{b}$ to $(2+(2 / 3)) E_{b}$. The proposed scheme deactivates the transmit antenna that is associated with the lowest channel gain and equally allocates the total transmit power to the other transmit antennas so that the statistical properties of the resulting effective channels are changed, and a certain degree of transmit diversity is achieved, similar to the classic antenna selection schemes [16].

\section{Cooperative ST-SSK Design}

The proposed ST-SSK is designed to achieve full diversity gain. However, we also note that the following potential problems may be encountered.

1) A high ST-SSK throughput only becomes achievable at the cost of employing a high number of transmit antenna elements, which is impractical for shirt-pocket-sized handheld devices.

2) A shadowing-induced power loss may be imposed on the multiple-antenna-aided transmitter, which results in a significant performance degradation at the destination.

To mitigate these potential problems, we invoke the proposed scheme for achieving cooperative diversity [17], [18], where multiple 


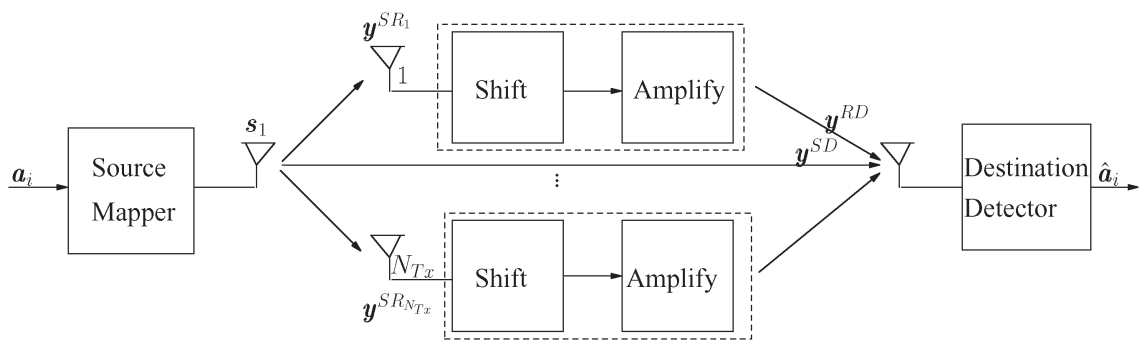

Fig. 2. AF-relaying-aided ST-SSK scheme with $N_{T x}$ relays to aid the SN.

single-element relay nodes (RNs) may share their antenna elements to form a virtual antenna array (VAA). We propose an AF-relaying-aided ST-SSK scheme (see Fig. 2), where the signal processing at the RNs exhibits a low complexity, because our ST-SSK design dispenses with interantenna synchronization.

Let us now consider the ST-SSK mapping function $f_{s}\left(t_{l}, \boldsymbol{a}_{i}\right)$ for scenarios with an even number of time slots. According to the mapping function $f_{s}\left(t_{l}, \boldsymbol{a}_{i}\right)$, the antennas are activated one by one to transmit the same signal. Therefore, the symbol vector that is transmitted at the $n$th $\left(1 \leq n \leq N_{T x}\right)$ antenna is equivalent to a right circular shift of the symbol vector that is transmitted by the $(n-1)$ th antenna.

Against this background, we specify the mapping function that is dedicated to the $n$th antenna element as $\left\{f_{s}^{n}\left(t_{l}, \boldsymbol{a}_{i}\right)\right\}_{n=1}^{N_{T x}}$, where the information bits are mapped to the $n$th antenna element according to $f_{s}\left(t_{l}, \boldsymbol{a}_{i}\right)$, except that the other $\left(N_{T x}-1\right)$ antenna elements that are indicated in $f_{s}\left(t_{l}, \boldsymbol{a}_{i}\right)$ are ignored. We arrange for the singleantenna-element-aided source node (SN) to perform the bit-to-antenna mapping according to $f_{s}^{1}\left(t_{l}, \boldsymbol{a}_{i}\right)$. The $r$ th $\left(1 \leq r \leq N_{T x}\right) \mathrm{RN}$ first performs a right circular shift of the received signal by $(r-1)$ positions and then activates the amplify and retransmit functions so that the ST-SSK mapping function $f_{s}\left(t_{l}, \boldsymbol{a}_{i}\right)$ may be completed by the $N_{T x}$ RNs in a distributed manner.

More explicitly, during the first transmission period, which is also referred to as the broadcast interval, the received signal at the destination node $(\mathrm{DN})$ is represented by

$$
\mathbf{y}^{S D}=\sqrt{P_{S}} \mathbf{h}^{S D}+\mathbf{n}^{S D}
$$

where $P_{S}$ denotes the transmit power at the $\mathrm{SN}$, whereas the $L$-element Rayleigh fading vector $\mathbf{h}^{S D}=\left[h_{f_{s}^{1}\left(t_{1}, a_{i}\right)}^{S D}, \ldots\right.$, $\left.h_{f_{S}^{1}\left(t_{L}, \boldsymbol{a}_{i}\right)}^{S D}\right]^{T}$ has a zero mean and a variance of $\sigma_{S D}^{2}$. The received signal vector $\mathbf{y}^{S D}$ has $L$ elements, whereas the AWGN vector $\mathbf{n}^{S D}$ has the same size and a variance of $N_{0}^{D}$.

Similarly, the signal that is received at the $r$ th $\mathrm{RN}$ may be represented by

$$
\mathbf{y}^{S R_{r}}=\sqrt{P_{S}} \mathbf{h}^{S R_{r}}+\mathbf{n}^{S R_{r}}
$$

where the $L$-element vector $\mathbf{h}^{S R_{r}}=\left[h_{f_{s}^{1}\left(t_{1}, a_{i}\right)}^{S R_{r}}, \ldots, h_{f_{s}^{1}\left(t_{L}, \boldsymbol{a}_{i}\right)}^{S R_{r}}\right]^{T}$ has a source-relay (SR) channel output power of $\sigma_{S R}^{2}$, whereas the $L$-element AWGN vector $\mathbf{n}^{S R_{r}}$ has a zero mean and a variance of $N_{0}^{R}$.

The $L$-element received signal vector $\mathbf{y}^{S R_{r}}$ should be right circularly shifted by $(r-1)$ positions and then amplified by a factor of $\alpha=\sqrt{P_{R} /\left(P_{S} \sigma_{S D}^{2}+N_{0}^{R}\right)}$, where $P_{R}$ denotes the transmit power at the RNs. The retransmitted signal at the $r$ th $\mathrm{RN}$ is then formulated as

$$
\overline{\mathbf{y}}^{S R_{r}}=\alpha\left(\sqrt{P_{S}} \overline{\mathbf{h}}^{S R_{r}}+\overline{\mathbf{n}}^{S R_{r}}\right)
$$

where the fading channel vector becomes $\overline{\mathbf{h}}^{S R_{r}}=\left[h_{f_{s}^{r}\left(t_{1}, \boldsymbol{a}_{i}\right)}^{S R_{r}}, \ldots\right.$, $\left.h_{f_{s}^{r}\left(t_{L}, \boldsymbol{a}_{i}\right)}^{S R_{2}}\right]^{T}$. It is shown that the ST-SSK mapping function $f_{s}\left(t_{l}, \boldsymbol{a}_{i}\right)$ is therefore formed at the $N_{T x}$ RNs using low-complexity signal processing. Furthermore, the total power that is shared by the SN and the RNs should now be normalized as

$$
P_{S}+P_{R}=\frac{m E_{b}}{2 L} .
$$

In the second transmission period, which is also referred to as the cooperation interval, the signal that is received at the $\mathrm{DN}$ is given by

$$
\begin{aligned}
\mathbf{y}^{R D} & =\sum_{r=1}^{N_{T x}} \overline{\mathbf{y}}^{S R_{r}} h^{R D_{r}}+\mathbf{n}^{R D} \\
& =\sum_{r=1}^{N_{T x}} \alpha\left(\sqrt{P_{S}} \overline{\mathbf{h}}^{S R_{r}}+\overline{\mathbf{n}}^{S R_{r}}\right) h^{R D_{r}}+\mathbf{n}^{R D} \\
& =\alpha \sqrt{P_{S}} \overline{\mathbf{h}}^{S R-R D}+\overline{\mathbf{n}}^{R D}
\end{aligned}
$$

where the $L$-element equivalent fading channel vector becomes $\quad \overline{\mathbf{h}}^{S R-R D}=\left[\bar{h}_{f_{s}\left(t_{1}, \boldsymbol{a}_{i}\right)}^{S R-R D}, \ldots, \bar{h}_{f_{s}\left(t_{L}, \boldsymbol{a}_{i}\right)}^{S R-R D}\right]^{T}=$ $\left[\sum_{r=1}^{N_{T x}}\left(h^{S R_{r}} h^{R D_{r}}\right)_{f_{s}\left(t_{1}, \boldsymbol{a}_{i}\right)}, \ldots, \sum_{r=1}^{N_{T x}}\left(h^{S R_{r}} h^{R D_{r}}\right)_{f_{s}\left(t_{L}, \boldsymbol{a}_{i}\right)}\right]^{T}$, whereas the equivalent noise matrix $\overline{\mathbf{n}}^{R D}=\sum_{r=1}^{N_{T x}} \alpha \overline{\mathbf{n}}^{S R_{r}} h^{R D_{r}}+$ $\mathbf{n}^{R D}$ has a variance of $\alpha^{2} \sigma_{R D}^{2} N_{0}^{R}+N_{0}^{D}$, where $\sigma_{R D}^{2}$ denotes the power of the fading channel between the RNs and the DN. It is shown that the noise at the RNs has also been amplified and forwarded to the DN.

Finally, the transmitted sequence $\hat{\boldsymbol{a}}_{i}$ is determined at the DN according to

$$
\begin{aligned}
\hat{\boldsymbol{a}}_{i}=\arg \min _{\boldsymbol{a}_{i}} \sum_{l}^{L} \mid y_{t_{l}}^{S D}- & \left.\sqrt{P_{S}} h_{f_{s}^{1}\left(t_{l}, \boldsymbol{a}_{i}\right)}^{S D}\right|^{2} \\
& +\sum_{l}^{L}\left|y_{t_{l}}^{R D}-\alpha \sqrt{P_{S}} \bar{h}_{f_{s}\left(t_{l}, \boldsymbol{a}_{i}\right)}^{S R-R D}\right|^{2} .
\end{aligned}
$$

\section{Simulation Results}

The uncoded BER versus the $E_{b} / N_{0}$ performance of a system with $N_{T x}=4, N_{R x}=1$ antennas and using the proposed open-loop ST-SSK modulation scheme discussed in Section III-A is characterized in Fig. 3. When the transmission time duration $L$ increases from 1 to 3 , the achievable throughput decreases from 2 (bits/symbol) to 2/3 (bits/symbol). At the same time, the achievable transmit diversity order increases from one to three, which is illustrated in Fig. 3 by the increased slope of the BER curves. Moreover, when the number of time slots increases to $L=4$, the slope of the BER curves remains the same as the slope of $L=3$, which demonstrated that the maximum 


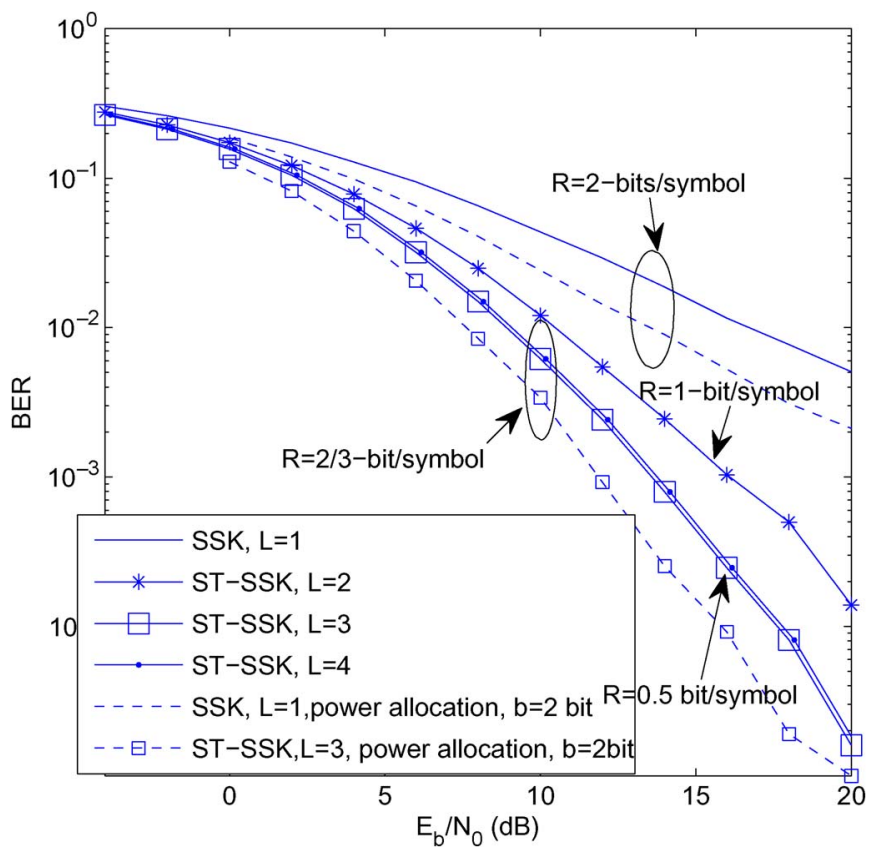

Fig. 3. Uncoded BER versus $E_{b} / N_{0}$ for $N_{T x}=4, N_{R x}=1$ for the following three approaches: 1 ) the ST-SSK scheme that uses $L=1 / 2 / 3$, respectively, and the shift-swap bit-to-antenna mapping $f_{s-w}$, as shown in Table I(b);2) the ST-SSK scheme that is combined with feedback-aided power allocation; and 3) the original SSK modulation.

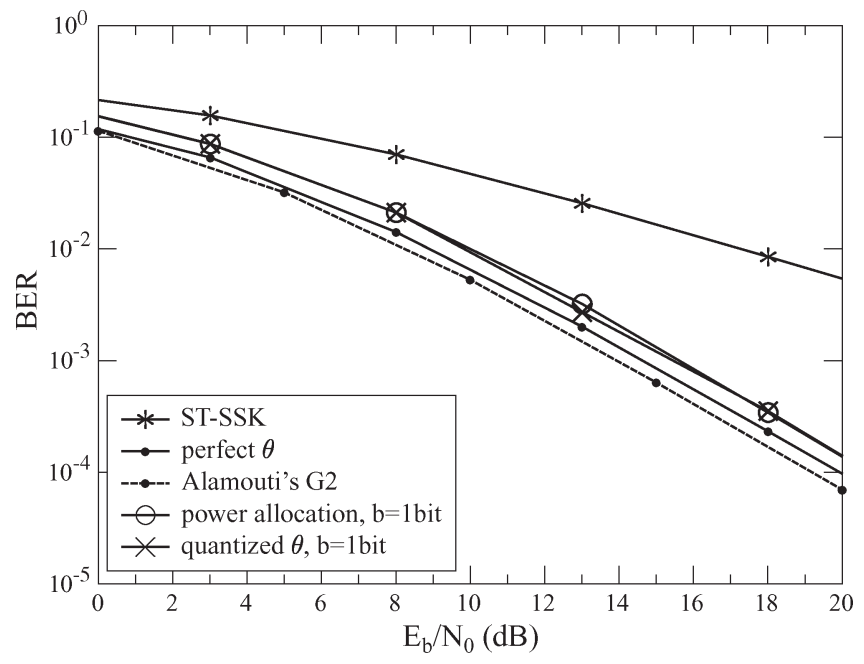

Fig. 4. Uncoded BER versus $E_{b} / N_{0}$ for ST-SSK with $N_{T x}=2, N_{R x}=1$ using perfect phase feedback, 1-bit quantized phase feedback, and 1-bit power allocation feedback aided, respectively. The performance of the original openloop ST-SSK modulation is also included for comparison.

achievable diversity order of an ST-SSK-modulation-aided system is three $\left(N_{T x}-1=3\right)$. Further BER improvements can be achieved by combining the ST-SSK scheme with the feedback-aided power allocation in Section III-C.

Fig. 4 characterizes the uncoded BER versus the $E_{b} / N_{0}$ performance for a throughput of $R=1$ (bits/symbol) and $N_{T x}=2$, $N_{R x}=1$. The following four scenarios are compared:

1) perfect phase feedback;

2) 1-bit feedback-aided phase rotation;

3) 1-bit feedback-aided power allocation;

4) the original open-loop SSK modulation.

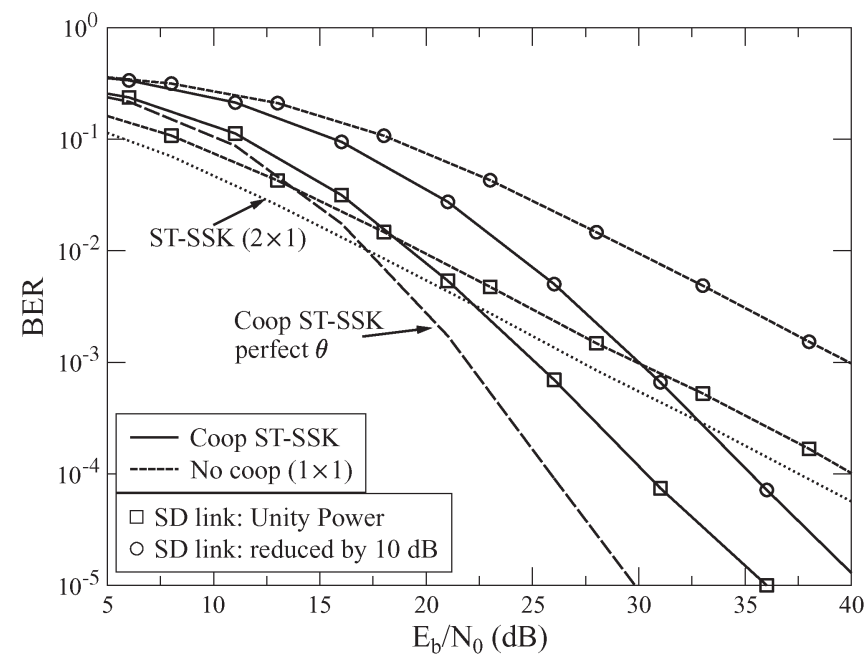

Fig. 5. Uncoded BER versus $E_{b} / N_{0}$ for the $N_{T x}=L=2$ AF-relayingaided ST-SSK scheme, where the transmit power at the SN and the RNs are normalized as $P_{S}=P_{R}=1 / 2$. The unit channel power is assumed for all links, i.e., $\sigma_{S R}^{2}=\sigma_{R D}^{2}=\sigma_{S D}^{2}=1$. The performance of the direct transmission without relaying and of the original ST-SSK scheme are also included for comparison.

For our proposed ST-SSK scheme, a diversity order of two results in a significant power gain, which is shown in Fig. 4. Hence, we observe that our ST-SSK scheme that relies on perfect phase feedback approaches the performance of the Alamouti G2 STBC. Moreover, using as few as a single bit to quantize the feedback phase information imposes only a modest performance degradation of about $1 \mathrm{~dB}$. The achievable BER performance that is recorded for using a 1-bit feedback for either phase information or power allocation is similar to one another.

The uncoded BER versus $E_{b} / N_{0}$ performance of our AF-relayingaided ST-SSK with $N_{T x}=L=2$ is shown in Fig. 5. A unit channel power is assumed for both the source-relay (SR) and the relay-destination (RD) links, as well as for the source-destination (SD) link, i.e., $\sigma_{S R}^{2}=\sigma_{R D}^{2}=\sigma_{S D}^{2}=1$. It is shown in Fig. 5 that the cooperative diversity provides a significant performance improvement over both the direct transmission regime and its ST-SSK counterpart. If a perfect phase feedback is available for the two SR links and the two RD links, the performance of the cooperative ST-SSK scheme is further improved, which is demonstrated in Fig. 5. Moreover, assuming that a 10-dB power loss occurred for the SD link due to a deep shadow fade, Fig. 5 shows that the performance degradation of the AF relaying scheme is only $5 \mathrm{~dB}$ at a BER of $10^{-5}$, because the SD links only contribute half the overall diversity order in our proposed cooperative ST-SSK scheme.

When using pilot-assisted channel estimation, the resulting estimated channel may be formulated as $\hat{\boldsymbol{h}}=\sqrt{h_{e}} \boldsymbol{g}+\boldsymbol{h}$, where $h_{e}$ represents the variance of the channel estimation error and the vector $\boldsymbol{g}$ is constituted by $N_{T x}$-dimensional random Gaussian variables with a zero mean and unit variance, whereas the $N_{T x}$-element vector $\boldsymbol{h}$ represents the actual CIR. The coded BER performance versus the variance $h_{e}$ of the channel estimation error recorded for the various transmission schemes is illustrated in Fig. 6, where a rate $r_{c}=1 / 3$ convolutional code with a constraint length of seven is employed. It is demonstrated in Fig. 6(a) that, for $N_{T x}=2$, the original open-loop SSK scheme is sensitive to channel estimation errors. The proposed closed-loop scheme that uses the phase information and the feedbackaided power allocation regimes are both less sensitive to small channel estimation errors, e.g., to $h_{e}<0.05$. However, the sensitivity to high channel estimation errors becomes similar for all three schemes. It 


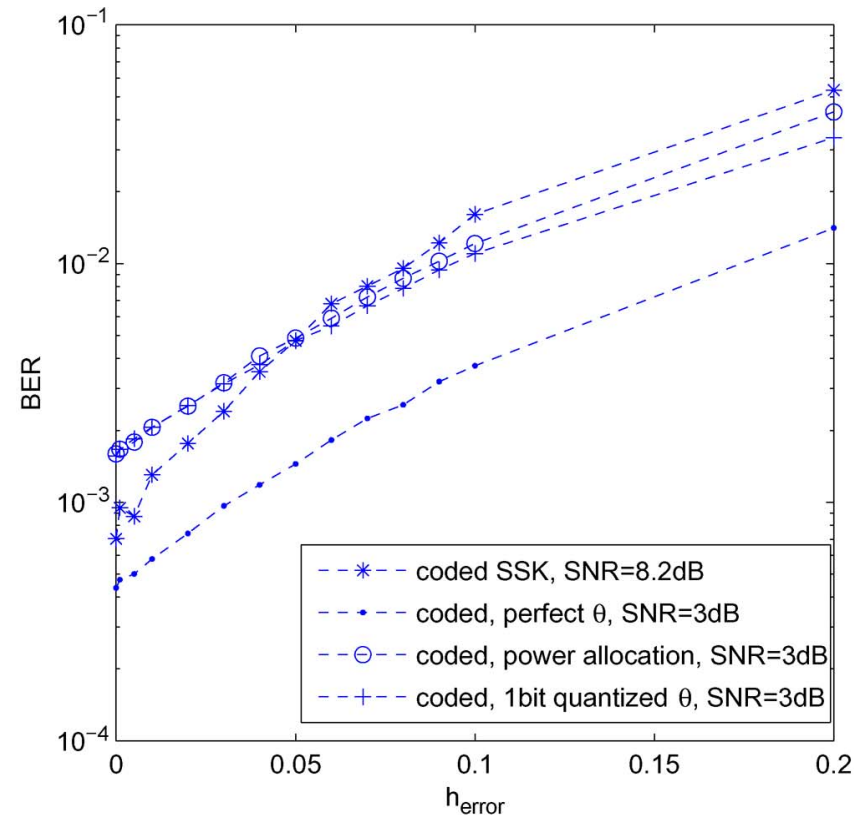

(a)

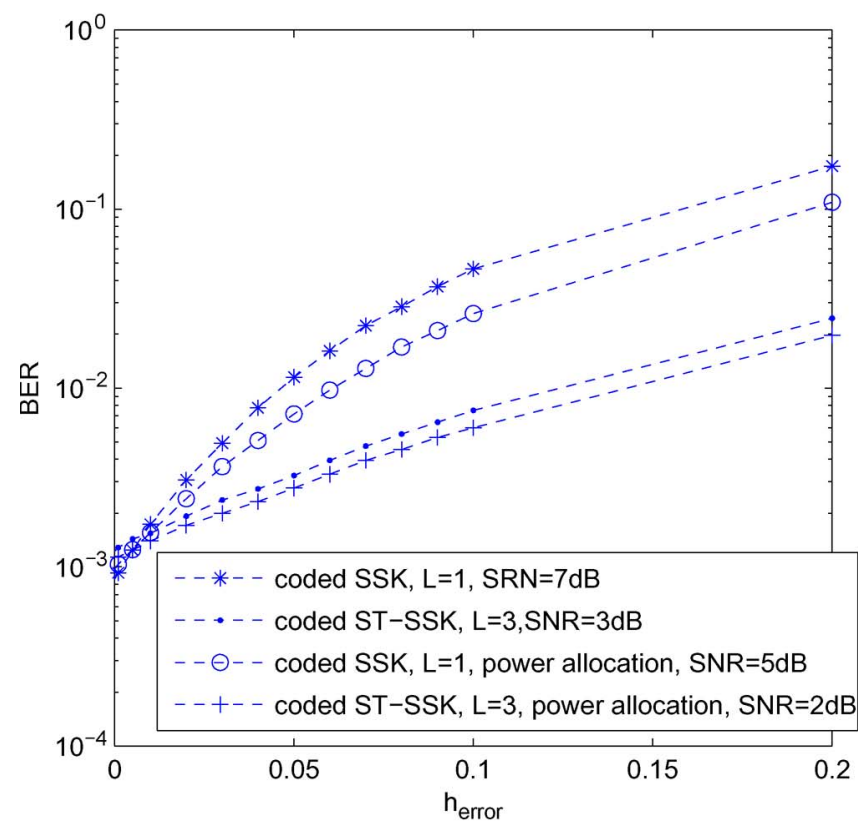

(b)

Fig. 6. Coded BER performance versus the variance of the channel estimation error $h_{e}$ at a fixed SNR per bit value using the following five approaches: 1) the original open-loop SSK modulation; 2) the proposed closed-loop scheme that uses perfect and quantized phase feedback for $N_{T x}=2 ; 3$ ) the proposed feedback-aided power allocation scheme for $N_{T x}=2 ; 4$ ) the proposed openloop ST-SSK scheme for $N_{T x}=4$; and 5) a combination of ST-SSK and feedback-aided power allocation for $N_{T x}=4$. (a) Coded $N_{T x}=2$. (b) Coded $N_{T x}=4$.

is also demonstrated in Fig. 6(b) that, for small channel estimation errors, the proposed ST-SSK scheme is significantly less sensitive than the original SSK scheme. Moreover, the employment of the proposed feedback-aided power allocation can reduce the sensitivity to channel estimation errors, provided that these errors are confined to relatively small values. However, the sensitivity to high channel estimation errors becomes similar for all three schemes. The reason for these observations is that the value of $\Delta h$ has been increased by using the proposed schemes. As a result, the proposed schemes became robust to small channel estimation errors.

\section{CONCLUSION}

In this paper, the following four novel schemes have been proposed:

1) the open-loop ST-SSK modulation, which is designed for systems with more than two transmit antennas;

2) the closed-loop feedback-aided phase rotation, which is conceived for systems with two transmit antennas;

3) the closed-loop feedback-assisted power allocation;

4) the AF-relaying-aided ST-SSK scheme, which is designed to achieve a useful transmit diversity for SSK modulation.

The proposed ST-SSK schemes significantly improved the achievable uncoded BER performance over independent and identically distributed (i.i.d.) Rayleigh fading channels. Moreover, the proposed cooperative ST-SSK scheme eliminates the practical problem of employing a high number of transmit antennas while simultaneously mitigating the performance degradation caused by shadow fading.

\section{REFERENCES}

[1] J. Jeganathan, A. Ghrayeb, L. Szczecinski, and A. Ceron, "Space-shift keying modulation for MIMO channels," IEEE Trans. Wireless Commun., vol. 8, no. 7, pp. 3692-3703, Jul. 2009.

[2] Y. A. Chau and S. H. Yu, "Space modulation on wireless fading channels," in Proc. IEEE 54th Veh. Technol. Conf., 2001, vol. 3, pp. 1668-1671.

[3] R. Y. Mesleh, H. Haas, S. Sinanovic, C. W. Ahn, and S. Yun, "Spatial modulation," IEEE Trans. Veh. Technol., vol. 57, no. 4, pp. 2228-2241, Jul. 2008.

[4] R. Mesleh, H. Haas, C. W. Ahn, and S. Yun, "Spatial modulation-A new low-complexity spectral-efficiency-enhancing technique," in Proc. 1st Int. Conf. Commun. Netw. China, Oct. 2006, pp. 1-5.

[5] G. J. Foschini, "Layered space-time architecture for wireless communication in fading environments when using multiple antennas," Bell Labs Tech. J., vol. 2, pp. 41-59, 1996.

[6] S. M. Alamouti, "A simple transmit diversity technique for wireless communications," IEEE J. Sel. Areas Commun., vol. 16, no. 8, pp. 1451-1458, Oct. 1998.

[7] L. Hanzo, M. Munster, B. J. Choi, and T. Keller, OFDM and MC-CDMA for Broadband Multiuser Communications, WLANs and Broadcasting. Hoboken, NJ: Wiley, 2003.

[8] L. Hanzo, J. S. Blogh, and S. Ni, 3G, HSDPA, HSUPA and FDD Versus TDD Networking: Smart Antennas and Adaptive Modulation. New York: Wiley/IEEE Press, 2008.

[9] M. Tsai and S. Yousefi, "A new iterative joint detection and decoding algorithm for V-BLAST architecture," IEEE Signal Process. Lett., vol. 16, no. 10, pp. 905-908, Oct. 2009.

[10] S. Sugiura, S. Chen, and L. Hanzo, "Reduced-complexity iterative Markov chain MBER detection for MIMO systems," IEEE Signal Process. Lett., vol. 16, no. 3, pp. 160-163, Mar. 2009.

[11] M. Renzo and H. Haas, "Improving the performance of space-shift keying (SSK) modulation via opportunistic power allocation," IEEE Commun. Lett., vol. 14, no. 6, pp. 500-502, Jun. 2010.

[12] J. Jeganathan, A. Ghrayeb, and L. Szczecinski, "Spatial modulation: Optimal detection and performance analysis," IEEE Commun. Lett., vol. 12, no. 8, pp. 545-547, Aug. 2008.

[13] C. Yuen, Y. L. Guan, and T. T. Tjhung, "Power-balanced orthogonal space-time block code," IEEE Trans. Veh. Technol., vol. 57, no. 5, pp. 3304-3309, Sep. 2008.

[14] C. Yuen, Y. Guan, and T. T. Tjhung, "Single-symbol-decodable differential space-time modulation based on QO-STBC," IEEE Trans. Wireless Commun., vol. 5, no. 12, pp. 3329-3334, Dec. 2006.

[15] C. Yuen, Y. Guan, and T. Tjhung, "Unitary differential space-time modulation with joint modulation," IEEE Trans. Veh. Technol., vol. 56, no. 6, pp. 3937-3944, Nov. 2007.

[16] R. S. Blum and J. H. Winters, "On optimum MIMO with antenna selection," IEEE Commun. Lett., vol. 6, no. 8, pp. 322-324, Aug. 2002.

[17] J. Laneman and G. Wornell, "Distributed space-time-coded protocols for exploiting cooperative diversity in wireless networks," IEEE Trans. Inf. Theory, vol. 49, no. 10, pp. 2415-2425, Oct. 2003.

[18] A. Sendonaris, E. Erkip, and B. Aazhang, "User cooperation diversity-Part I: System description," IEEE Trans. Commun., vol. 51, no. 11, pp. 1927-1938, Nov. 2003. 